\title{
Effect of Spinning Magnetic Ferrite on the Exclusion Zone
}

\author{
Yi Zhou1, Xing Wen², Alexandr A. Shpilman³, Quansheng Ren ${ }^{1 *}$ \\ ${ }^{1}$ Department of Electronics, Peking University, Beijing, China \\ ${ }^{2}$ School of Life Sciences, Peking University, Beijing, China \\ ${ }^{3}$ Health of the Kazakhstan's People Ltd., Karaganda, Kazakhstan \\ Email: *qsren@pku.edu.cn
}

How to cite this paper: Zhou, Y., Wen, X., Shpilman, A.A. and Ren, Q.S. (2017) Effect of Spinning Magnetic Ferrite on the Exclusion Zone. Journal of Biosciences and Medicines, 5, 1-7.

https://doi.org/10.4236/jbm.2017.512001

Received: June 26, 2017

Accepted: November 18, 2017

Published: November 21, 2017

\begin{abstract}
Colloidal and molecular solutes in suspension are excluded from the vicinity of various hydrophilic surfaces, leaving an exclusion zone (EZ) of hundreds of micrometers. Such exclusion zones were observed in the vicinity of many types of surface including artificial and natural hydrogels, biological tissues as well as hydrophilic polymers. Old studies have shown that the near-surface exclusion zone expands extensively in the presence of incident radiant energy, especially the infrared light. Developments of electromagnetic biology and quantum biology indicate that electron and nuclear spin may have a direct impact on the biological process. Inspired by these researches, the effects of spinning magnetic ferrite on EZ were studied. It is shown that spinning magnetic ferrite profoundly expands these zones in an irreversible manner.
\end{abstract}

\section{Keywords}

Magnetic Ferrite Rotator, Exclusion Zone, Hydrophilic Surface,

Radiant Energy

\section{Introduction}

The exclusion zone (EZ) of water develops in the direct vicinity of hydrophilic materials [1]. When water interacts with hydrophilic surfaces, the interfacial water molecules reorder, which cause a zone devoid of microscopic particles. The size of EZ changes with different hydrophilic materials, ranging from tens to hundreds of micrometers [2]. The water of the EZ, also named the fourth phase water, has been proposed to be in a liquid crystal state, beyond solid, liquid, and vapor. This interfacial area, negatively charged, is more ordered than bulk water [3], and its physical properties are much different. On the other hand, as a hy- 
drophilic interface, phospholipid bilayer membranes are widely found in organisms. Much of the water in the cell is very near to these hydrophilic surfaces and therefore is structured water. The latest research shows that water's role in biology may be central. Albert Szent-Gyorgyi, who won the Nobel Prize for discovering vitamin $\mathrm{C}$, has described this centrality as "Life is water dancing to the tune of solids [4]."

Quantum biology studies have shown that electron and nuclear spin plays important roles in many life processes, such as long-range electron transfer, the oxidation of water, and neural processing [5]. Particularly, it is shown that chirality and spin may play several important roles in biology, which have long been ignored [6]. In the field of water research, previous studies only considered the effects of radiant energy on EZ. The relationship between spin and EZ has not been investigated.

In the present study, we utilize a nickel-manganese ferrite rotator designed by Alexander A. Shpilman [7], which is presumed to generate a spin field arising from electron spin. We investigated the effects of spinning magnetic ferrite on EZ size, and found that the presumed spin field can promote the growth of the exclusion zone. This paper revealed the relationship between the so-called spin field relating with electron spin and the formulation of structured water near hydrophilic membrane. Considering the universality of electron spin and structured water in the organisms, our study may be of significance for quantum biology research.

\section{Experimental}

\subsection{General Outline}

As a hydrophilic substance, Nafion 117 per-fluorinated membrane was used in the experiments. Nafion is a perfluorosulfonic ionomer that consists of a polytetrafluoroethylene backbone and regularly spaced side chains terminated by sulfonate ionic groups [8]. Carboxylate microspheres, 2 microns, were used to show the extent of the exclusion zone. Generally, the microspheres were stored at $4^{\circ} \mathrm{C}$ atmospheres when not been used [9].

The nickel-manganese ferrite rotator used in experiments was designed by Alexander A. Shpilman (Figure 1(a)). Detailed dimension and structure are in Figure 1(b). The ferrite ring 2 rotates clockwise (viewed from above) around the axis 1 at a rate of several thousand revolutions per minute. $\mathrm{D}$ is a drive motor that provide energy for axis 1 . Silver cup 3 plays a role of a reflector. Cylindrical coil 4 creates axial vector potential and toroidal coil 6 creates radial vector potential. The superposition of vector potential in orthogonal directions forms a magnetic field of a spiral structure, which causes axial polarization of materials falling in area of its action. Iron cores 5, 8 are used to enhance the magnetic field.

All experiments were carried out at $20^{\circ} \mathrm{C}-23^{\circ} \mathrm{C}$ and in a dark room to minimize background noise. 


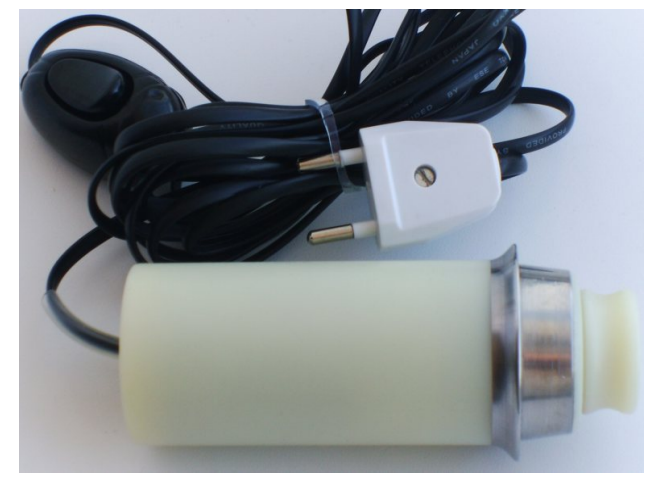

(a)

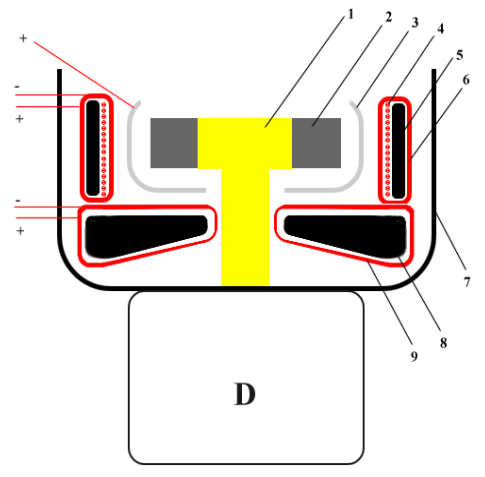

(b)

Figure 1. (a) The profile of the ferrite rotator; (b) The physical structure of the ferrite rotator.

\subsubsection{Nafion Pretreatment}

Nafion membranes were cut into $1 \mathrm{~cm} \times 2 \mathrm{~cm}$ squares. Peel off the thin backing layer, then dunk in water to separate the Nafion from the thick coversheet. All these square membranes were processed as follow steps: 1 hour in lightly boiling $\left(\sim 80^{\circ} \mathrm{C}\right) 5 \% \mathrm{H}_{2} \mathrm{O}_{2} 0.5$ hour in DI water 1 hour in lightly boiling $5 \% \mathrm{H}_{2} \mathrm{SO}_{4}$. At last, Store these membranes in DI water for experiments [10].

\subsubsection{Chamber Preparation}

To observe whether microscopes were excluded from the zones near the Nafion-117 surface, a chamber with floor was used. This chamber was made using 3D printing technology. It was a cuboid of length $5 \mathrm{~cm} \times$ width $2 \mathrm{~cm} \times$ height 1 $\mathrm{mm}$ with a hole in the center. The hole was a rectangle of length $3 \mathrm{~cm} \times$ width $1.6 \mathrm{~cm} \times$ height $1 \mathrm{~mm}$.

For sample illumination, an ordinary LED was used for experiment. After pretreatment, the Nafion membrane was attached to the medial surface of the chamber and illuminated by LED with the optical axis perpendicular to it. The chamber was filled with an aqueous suspension of microspheres and placed on a slide. The slide was viewed under an electron microscopy.

The ferrite rotator was used to investigate the effect of spin field on EZ size. The procedure of the experiment was the same as above in addition to replacing the LED with the ferrite rotor. Since the ferrite rotor can only work continuously for no more than 20 minutes at a time, it would be stopped for about 10minutes after working for 15 minutes. The distance between the ferrite rotor and the $\mathrm{Na}$ fion membranes are usually $20 \mathrm{~cm}$. The position of the ferrite rotor is slightly higher than the chamber so that the membranes were exposed directly to the field generated.

\subsection{Results}

Microspheres immediately translated away from the edges of the Nafion sheet, forming an exclusion zone of 250 um within 10 minutes. The width of the zone then grew more slowly at an almost unseen speed after 30 minutes. The results 
illustrated in Figure 2, show that there are not obvious changes between 25 min and $30 \mathrm{~min}$.

The effect of radiant energy on EZ size was very obvious. An ordinary LED was applied 40minutes after the establishment of the exclusion zone to ensure that the establishment of the EZ was stable. The EZ size began to increase, and expanded almost double times as the baseline EZ size after 120 minutes of exposure (Figures 2(a)-(d)). To minimize any effects of microscope illumination, the microscope lamp was turned off when the EZ was not been observed.

Similar with the experiments of radiant energy on Nafion membranes, ferrite rotor was applied when there were not obvious changes of EZ size. The baseline EZ size was selected after 30 minutes when the width was no longer significantly changed. Pause time of rotor were not been calculated. The microscope lamp was turned off when the EZ was not been observed. The width of EZ increased to about 1.5 times as the baseline EZ size after 60 minutes of exposure (Figures $3(\mathrm{a})-(\mathrm{d}))$

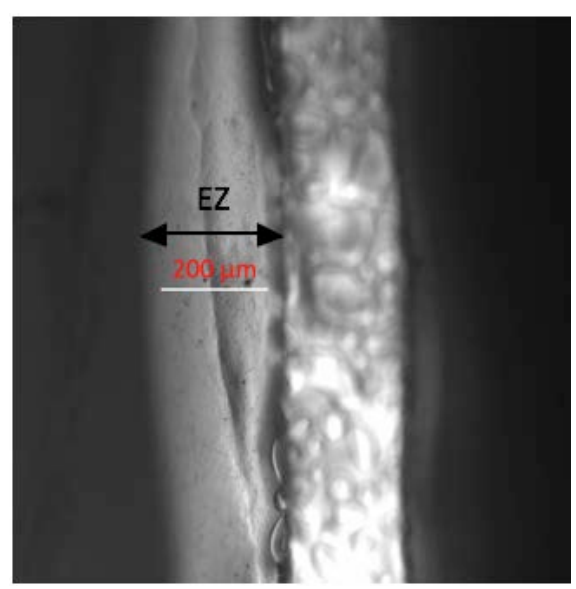

(a)

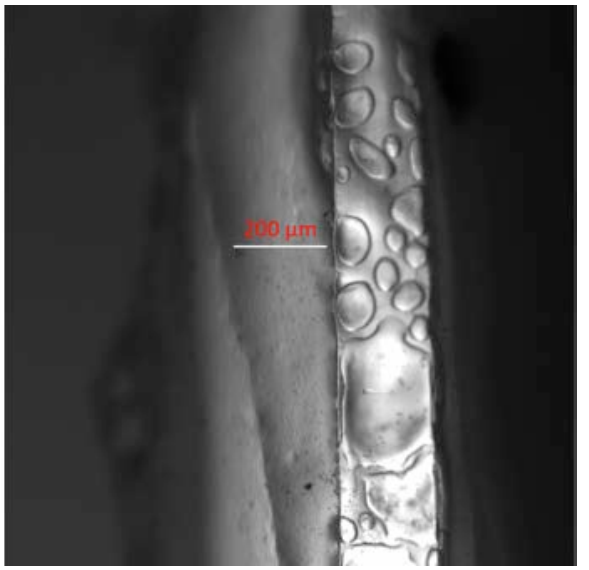

(c)

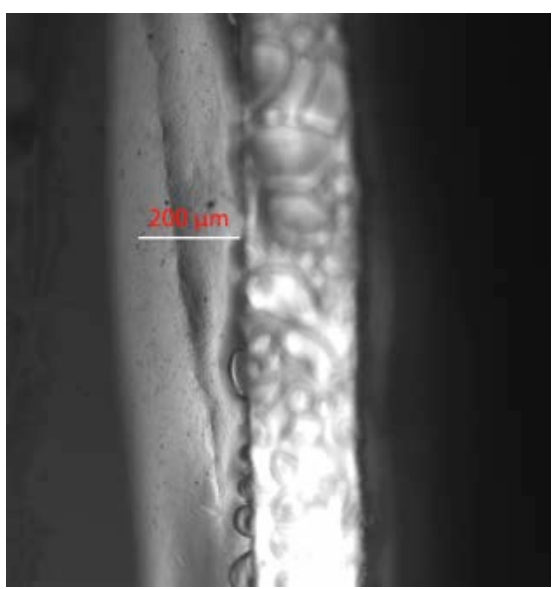

(b)

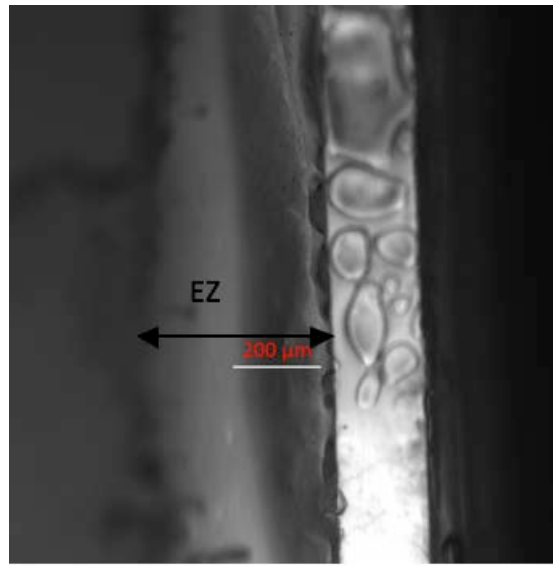

(d)

Figure 2. (a) 25 minutes in dark; (b) 30 minutes in dark; (c) 10 minutes of illumination; (d) 2 hours of illumination. The EZ size (d) expanded almost double times as the baseline ((a), (b)) EZ size after 120munites of exposure. 


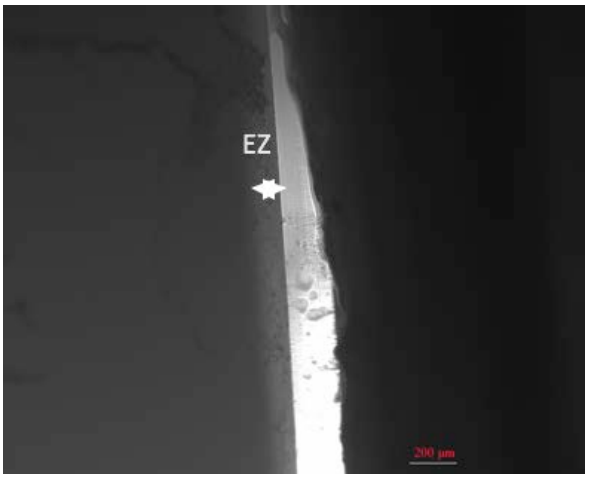

(a)

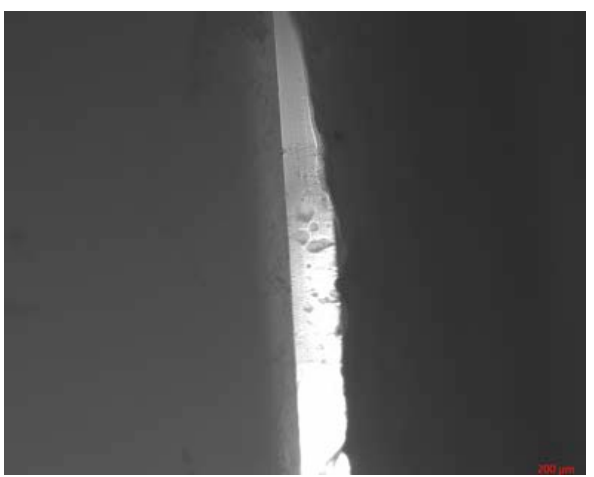

(b)

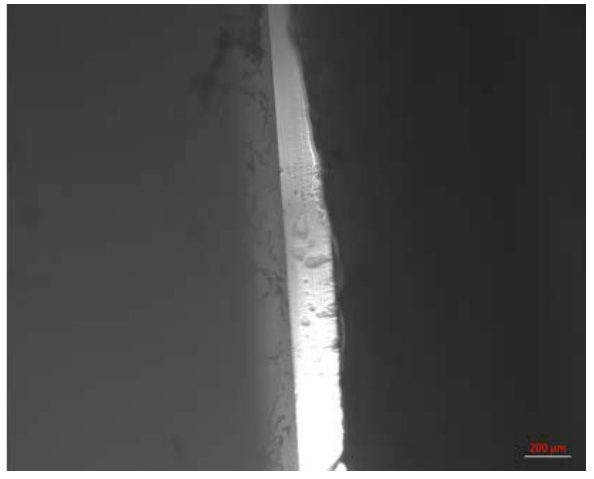

(c)

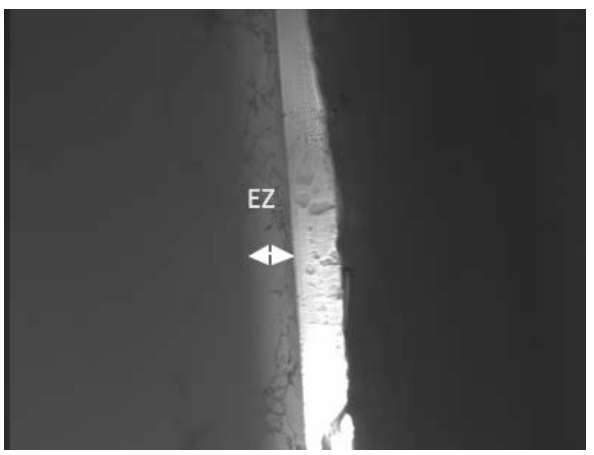

(d)

Figure 3. (a) 20 minutes in dark; (b) 30 minutes in dark; (c) 10 minutes irradiation of ferrite rotor; (d) 1 hour irradiation of ferrite rotor. The EZ size (d) expanded almost 1.5 times as the baseline ((a), (b)) EZ size after 60 munites of exposure. 


\section{Discussion}

Generally, it is hard to establish EZ with non-pretreatment nafion membranes. There is another simple method to form EZ without the special designed chamber. A membrane could be placed between two large glass cover slips. Microspheres solution was drooped along the edge of slips. However, EZ was unstable as there was only a small amount of microsphere solution which was often evaporated in a short time. The chamber was able to accommodate more solution, which was suitable for long observation.

A series of LEDS were used in the work of Binghua Chai [3]. An integrated holder was built to keep the pinhole and LED together as a single unit to maximize incident power. Growth of EZ was more obvious compared with our experiment. But radiant energy was proved to be helpful for EZ build up in a simpler way with an ordinary way of exposure.

The most important result of this study is that the width of EZ grows in the presence of a nickel-manganese ferrite rotator. Although the mechanism of exclusion is not yet clear, growth of this more ordered, negatively charged zone may be dependent on the presumed spin field relating with the circular rotating electron spin.

\section{Conclusion}

In conclusion, EZ was established using Nafion membranes which must be pretreated and the width expanded with Irradiation of an ordinary LED. This study reveals that the effects of the field generated by a ferrite rotator could promote the growth of exclusion zones. Considering the universality of electron spin and structured water in the organisms, our study may be of significance for quantum biology research.

\section{Acknowledgements}

This work is supported by National Natural Science Foundation of China (NSFC) (No. 61471010) and Dunhe Foundation.

\section{References}

[1] Zheng, J.-M., et al. (2006) Surfaces and Interfacial Water: Evidence That Hydrophilic Surfaces Have Long-Range Impact. Advances in Colloid and Interface Science, 127, 19-27. https://doi.org/10.1016/j.cis.2006.07.002

[2] Klyuzhin, I., Symonds, A., Magula, J. and Pollack, G. (2008) New Method of Water Purification Based on the Particle-Exclusion Phenomenon. Environmental Science \& Technology, 42, 6160-6166. https://doi.org/10.1021/es703159q

[3] Chai, B., Yoo, H. and Pollack, G. (2009) Effect of Radiant Energy on Near-Surface Water. Journal of Physical Chemistry B, 113, 13953-13958. https://doi.org/10.1021/jp908163w

[4] Pollack, G.H. (2010) Water, Energy and Life: Fresh Views from the Water's Edge. International Journal of Design \& Nature and Ecodynamics. A Transdisciplinary Journal Relating to Nature, Science and the Humanities, 5, 27-29. 
https://doi.org/10.2495/DNE-V5-N1-27-29

[5] Lambert, N., Chen, Y.N., Cheng, Y.C., Li, C.M., Chen, G.Y. and Nori, F. (2013) Quantum Biology. Nature Physics, 9, 10-18. https://doi.org/10.1038/nphys2474

[6] Michaeli, K., Kantor-Uriel, N., Naaman, R. and Waldeck, D.H. (2016) The Electron's Spin and Molecular Chirality-How Are They Related and How Do They Affect Life Processes? Chemical Society Reviews, 45. https://doi.org/10.1039/C6CS00369A

[7] Shpilman, A. (2010) The High-Frequency Three-Phase Generator. http://spinfield.idhost.kz/ALMANACH/3n00/ShemaA.htm

[8] O'Rourke, C., Klyuzhin, I., Park, J.S. and Pollack, G.H. (2011) Unexpected Water Flow through Nafion-Tube Punctures. Physical Review E, 83, Article ID: 056305. https://doi.org/10.1103/PhysRevE.83.056305

[9] Bunkin, N.F., Kozlov, V.A., Ignatiev, P.S., Suyazov, N.V., Shkirin, A.V. and Atakhodzhaev, I.A. (2012) Refraction Coefficient of Water and Aqueous Solutions in the Optical Frequency Range in the Vicinity of Nafion. Biophysics, 57, 733-749. https://doi.org/10.1134/S0006350912060048

[10] Fuller, T.F. and Newman, J. (1992) Experimental Determination of the Transport Number of Water in Nafion 117 Membrane. Journal of the Electrochemical Society, 139, 1332-1337. https://doi.org/10.1149/1.2069407 